\title{
A Research On Android Technology With New Version Naugat(7.0,7.1)
}

\author{
Nikhil M. Dongre, Tejas S. Agrawal, \\ Ass.prof. Sagar D. Pande \\ (Dept. CSE, Student of PRPCOE, SantGadge baba Amravati University, nikheeldongre@gmail.com \\ contact no: 8408895842 ) \\ (Dept. CSE, Student of PRMCEAM, SantGadge baba Amravati University, tejasagrawal146@ gmail.com \\ contact no: 9146951658)
}

(Dept. CSE, Assistant professor of PRPCOE, SantGadge baba Amravati University, sagarpande30@ gmail.com, contact no:9405352824)

\begin{abstract}
Android "Naugat" (codenamed Android $N$ in development) is the seventh major version of Android Operating System called Android 7.0. It was first released as a Android Beta Program build on March 9, 2016 with factory images for current Nexus devices, which allows supported devices to be upgraded directly to the Android Nougat beta via over-the-air update. Nougat is introduced as notable changes to the operating system and its development platform also it includes the ability to display multiple apps on-screen at once in a splitscreen view with the support for inline replies to notifications, as well as an OpenJDK-based Java environment and support for the Vulkan graphics rendering API, and "seamless" system updates on supported devices.
\end{abstract}

Keywords: jellybean, kitkat, lollipop, marshmallow, naugat

\section{Introduction}

This research has been done to give you the best details toward the exciting new frontier of open source mobile development. Android is the newest mobile device operating system, and this is one of the first research to help the average programmer become a fearless Android developer. The development of Android started in 2003 by Android, Inc., which was purchased by Google in 2005 [1].The version history of the Android mobile operating system began with the release of the Android alpha in November 5, 2007.

The first commercial version, Android 1.0, was released in September 2008. Android is continually developed by Google and the Open Handset Alliance, and it has seen a number of updates to its base operating system since the initial release.

Versions 1.0 and 1.1 were not released under specific code names, but since 2009's Android 1.5 Cupcake, Android versions have had confectionery-themed code names.A version of Android KitKat exclusive to Android Wear devices was released on June 25, 2014, with an API level of 20.Each is in alphabetical order, with the most recent major version being Android 7.0 Nougat, released in August 2016.[1]

\begin{tabular}{|c|c|c|c|c|}
\hline Code name & Version number & Initial release date & API level & Support status \\
\hline Alpha & 1.0 & September 23, 2008 & 1 & Unsupported \\
\hline Beta & 1.1 & February 9, 2009 & 2 & Unsupported \\
\hline Cupcake & 1.5 & April 27, 2009 & 3 & Unsupported \\
\hline Donut & 1.6 & September 15, 2009 & 4 & Unsupported \\
\hline Eclair & $2.0-2.1$ & October 26, 2009 & $5-7$ & Unsupported \\
\hline Froyo & $2.2-2.2 .3$ & May 20, 2010 & 8 & Unsupported \\
\hline Gingerbread & $2.3-2.3 .7$ & December 6, 2010 & $9-10$ & Unsupported \\
\hline Honeycomb & $3.0-3.2 .6$ & February 22, 2011 & $11-13$ & Unsupported \\
\hline Ice Cream Sandwich & $4.0-4.0 .4$ & October 18, 2011 & $14-15$ & Unsupported \\
\hline Jelly Bean & $4.1-4.3 .1$ & July 9, 2012 & $16-18$ & Unsupported \\
\hline KitKat & $4.4-4.4 .4$ & October 31,2013 & 19 & Unsupported \\
\hline Lollipop & $5.0-5.1 .1$ & November 12, 2014 & $21-22$ & Unsupported \\
\hline Marshmallow & $6.0-6.0 .1$ & October 5, 2015 & 23 & Supported \\
\hline Nougat & $7.0-7.1 .2$ & August 22, 2016 & $24-25$ & Supported \\
\hline
\end{tabular}




\section{Android System Architecture Details}

Most of the Android user are using their Android phone just for calls, SMS, browsing and basic apps, But form the development prospective, we should know about Android internal structure. Android uses several partitions (like boot, system, recovery, data etc) to organize files and folders on the device just like Windows OS. Each of these partitions has its own functionality, but most of us don't know the significance of each partition and its contents

There are mainly 6 partitions in Android phones, tablets and other Android devices. Below is the list of partition for Android File System. Note that there might be some other partitions available, it differs from Model to Model. But logically below 6 partitions can be found in any Android devices.[2]

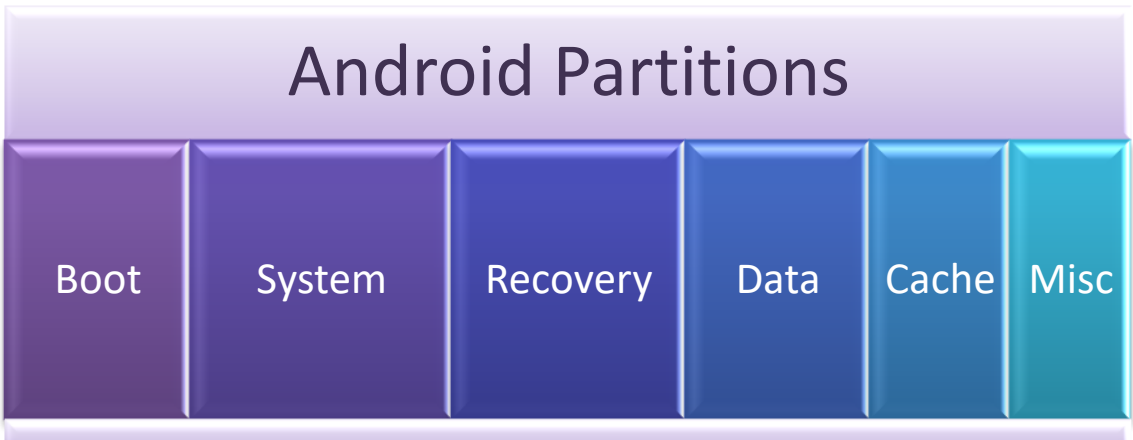

* About applications:

Fig.1 Android partitions

The first layer of the android archtecture is an application layer,in this layer your written application which to be installed are avilable on this layer only.Example like applications Contacts books,Browser,Games etc. are available in it.that shows the below figure:

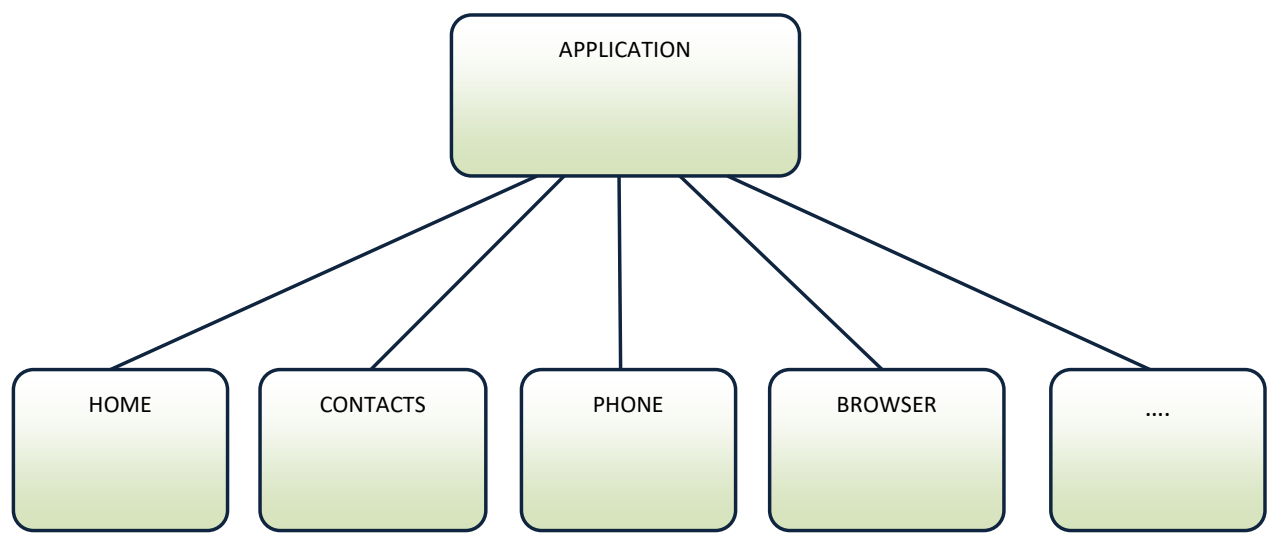

LEVEL-1 FIGURE

\section{* Application framework:}

This layer provides many high- level services to applications in the form of java classes.Applications developers are allowed to make use of these serveces in there applications.

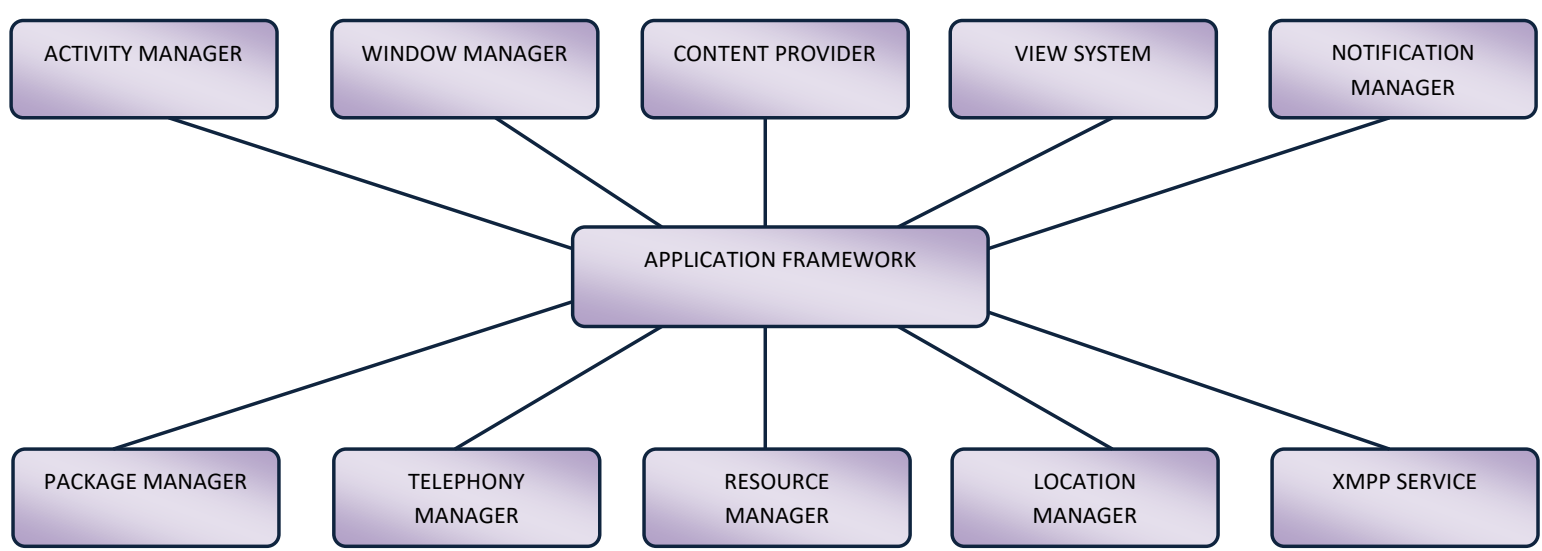

LEVEL - 2 FIGURE 


\section{- Android libraries:}

Android libraries are available on top of Linux kernel there is a set of libraries including open-source Web browser engine web kit, well known library libc, SqLite database which is a useful option for storage and sharing of application data, libraries to play and record and video ,SSL libraries responsible for internet security etc.

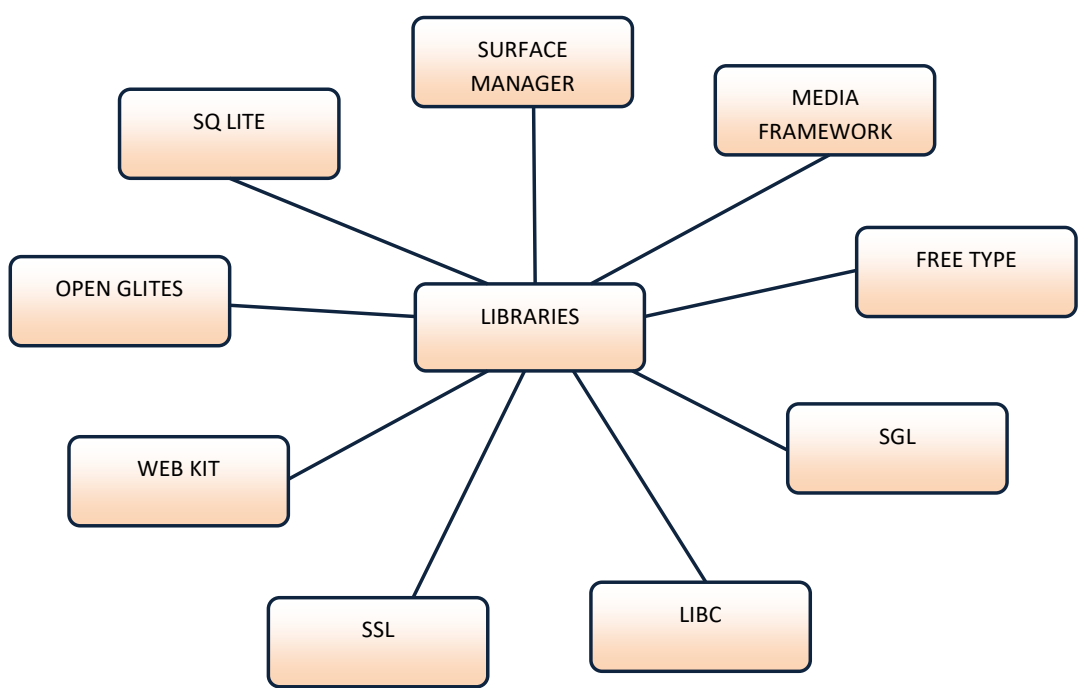

LEVEL - 3 FIGURE

\section{* Android Runtime system:}

This is the third part of android architecture this section provides a key component called Dalvik Virtual Machine specially designed and optimized for android. The Dalvik Virtual Machine makes use of Linux core features like memory management and multi-threaded, which is intrinsic in the java language. The Dalvik Virtual Machine enables every android application to run in its own process, with its own instance of the Dalvik Virtual Machine.

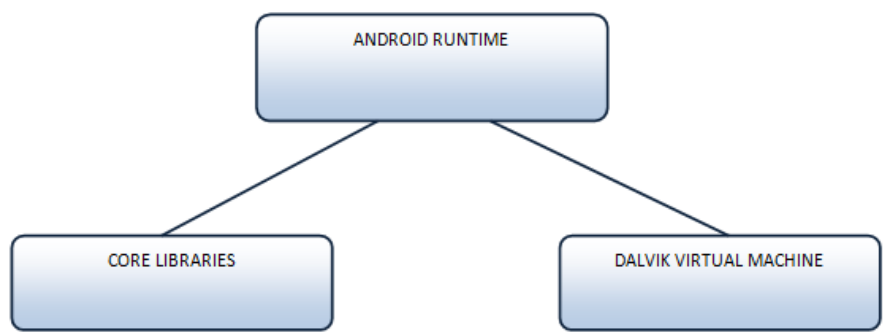

LEVEL - 4 FIGURE

\section{* Android Linux Kernel:}

In the Linux layers Linux 2.6 with approximately 115 patches. This provides basic system functionality like process management, memory management, device management like camera, keypad, display etc. also, the kernel handles all the things that Linux is really good such s networking and a vast array of device drivers, which take the pain out of interfacing to peripheral hardware.

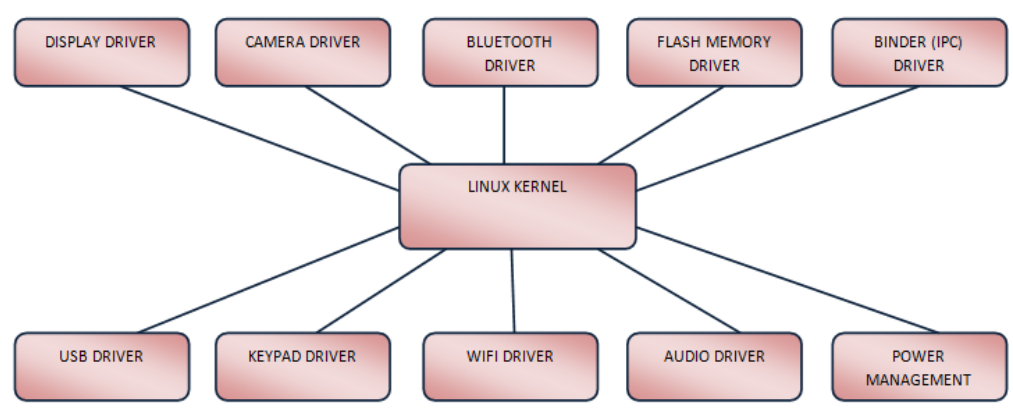

LEVEL - 5 FIGURE 


\section{History}

On March 9, 2016, ahead of the Google I/O developer conference, Google released the first beta of Android "N" as part of a new "Android Beta Program" intended for testing by developers and enthusiasts before official release "this summer". The developer preview builds were compatible with only current Google Nexus devices; the 5X, 6P, 6, 9, Pixel C, and Nexus Player. The "Android Beta Program" that was introduced allows testers to opt-in for over-the-air updates to new beta versions as they are released.[3]

On April 13, 2016, Android N Beta Preview 2 was released [4]. Google further discussed Android "N" during the I/O keynote on May 18, 2016, and unveiled its new virtual reality platform Daydream. Beta Preview 3 , the first preview release deemed suitable for wider public beta testing, was released at this time. Google also announced that it would hold a contest to determine the official release name of the operating system.[5][6]

Beta Preview 4 was released on June 15, 2016.[7] On June 30, 2016, Google announced that N's release name would be "Nougat"; it was also confirmed that Nougat would be version 7.0 of Android.[9] The final Beta Preview, 5, was released on July 18, 2016.[8]Android 7.0 was officially released on August 22, 2016, with the Nexus 6, Nexus 5X, Nexus 6P, Nexus 9, Nexus Player, Pixel C and General Mobile 4G as the first devices to receive the update.[10]

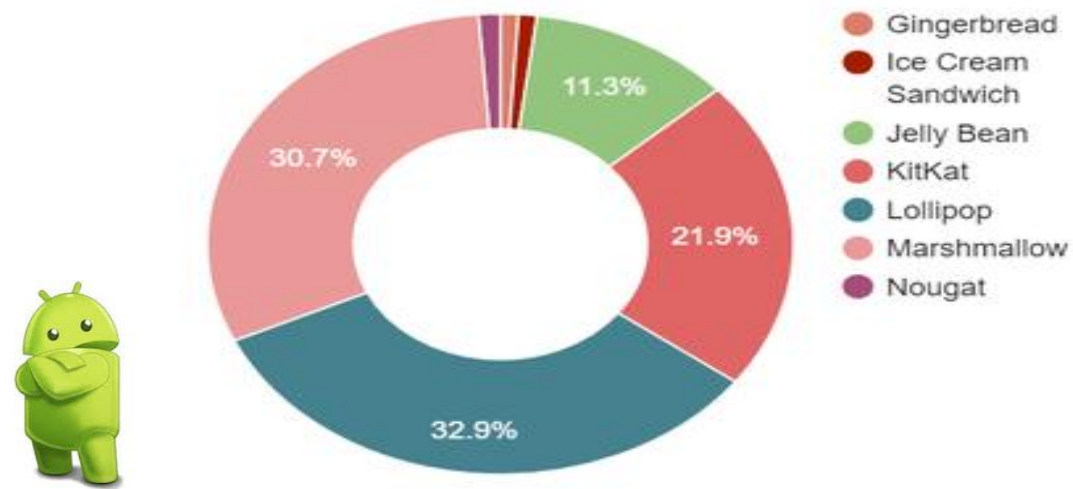

Fig. 2 Global Android version distribution as of February 2017

A post-release update known as Android 7.1 was pre-loaded onto Google's Pixel and Pixel XL smart phones released in October 2016; the new version adds support for the Google Daydream VR platform, image keyboards, expanded emoji support (including male and female versions of gendered emoji), support for actions to be displayed in menus on home screen app shortcuts, and other new features. A preview of 7.1 for existing Nexus devices was released via Android Beta Program later in the month,[11] and officially released as Android 7.1.1 on December 5, 2016.[12][13]

\section{- About previous versions of android:}

To overcome the drawbacks available in the previous versions of android operating system the new version is developed and introduced that is android nougat (7.0).this version of android overcomes all drawbacks of previous android versions, previous versions are explained with their features in below tables:

\section{- The Popular Versions those are by API level:}

$$
\text { Android 4.0 Ice Cream Sandwich (API 14) }
$$

The SDK for Android 4.0.1 (Ice Cream Sandwich), based on Linux kernel 3.0.1, was publicly released on October 19, 2011.

Google's Gabe Cohen stated that Android 4.0 was "theoretically compatible" with any Android 2.3.x device in production at

that time.[14] The source code for Android 4.0 became available on November 14, 2011. Ice Cream Sandwich was the last version to officially support Adobe Systems' Flash player. [15]The update introduced numerous new features[16]

\begin{tabular}{|c|c|}
\hline version to orsion :4.0, 4.0.1, 4.0.2 & Release date : November-2011-4,Oct-21, Nov-28
\end{tabular}

- Major refinements to the "Holo" interface with new Robot font family

- Soft buttons from Android 3.x are now available for use on phones

- Separation of widgets in a new tab, listed in a similar manner to applications, Easier-to-create folders, with a drag-and-drop style

- Improved visual voicemail with the ability to speed up or slow down voicemail messages, Pinch-to-zoom functionality in the calendar

- Integrated screenshot capture (accomplished by holding down the Power and Volume-Down buttons)

- Improved error correction on the keyboard, Ability to access applications directly from lock screen, Improved copy and paste functionality

- Better voice integration and continuous, real-time speech to text dictation

- Face Unlock, a feature that allows users to unlock handsets using facial recognition software, Automatic syncing of browser with users' Chrome bookmarks 
- Data Usage section in settings that lets users set warnings when they approach a certain usage limit, and disable data use when the limit is exceeded

- Ability to shut down applications from the recent apps list with a swipe.[17]

- Improved camera application with zero shutter lag, time lapse settings, panorama mode, and the ability to zoom while recording, Built-in photo editor

- New gallery layout, organized by location and person, Refreshed "People" application with social network integration, status updates and hi-res images

- Android Beam, a near-field communication feature allowing the rapid short-range exchange of web bookmarks, contact info, directions, YouTube videos and other data ,Support for the WebP image format ,Hardware acceleration of the UI ,Wi-Fi Direct[18][19]

- $\quad 1080 \mathrm{p}$ video recording for stock Android devices ,Android VPN Framework (AVF), and TUN (but not TAP) kernel module. Prior to 4.0, VPN software required rooted Android, Fixed minor bugs for the Samsung Galaxy Nexus,

- $\quad$ Fixed minor bugs on the Verizon Galaxy Nexus, the US launch of which was later delayed until December 2011

\begin{tabular}{|l|l|}
\hline \multicolumn{2}{|c|}{ Android 4.0.3 Ice Cream Sandwich (API 15) } \\
\hline \multicolumn{1}{|c|}{ Version : 4.0.3 4.0.4 } & \multicolumn{1}{c|}{ Release date : December-16,-2011, March 29, 2012} \\
\hline - & \multicolumn{1}{|c|}{ Fumerous bug fixes and optimizations, Improvements to graphics, databases, spell-checking and Bluetooth functionality ,New } \\
APIs for developers, including a social stream API in the Contacts provider, Calendar provider enhancements, \\
- New camera applications enhancing video stabilization and QVGA resolution ,Accessibility refinements such as improved \\
$\begin{array}{l}\text { content access for screen readers, Stability improvements, Better camera performance, Smoother screen rotation ,Improved phone } \\
\text { number recognition [20] }\end{array}$
\end{tabular}

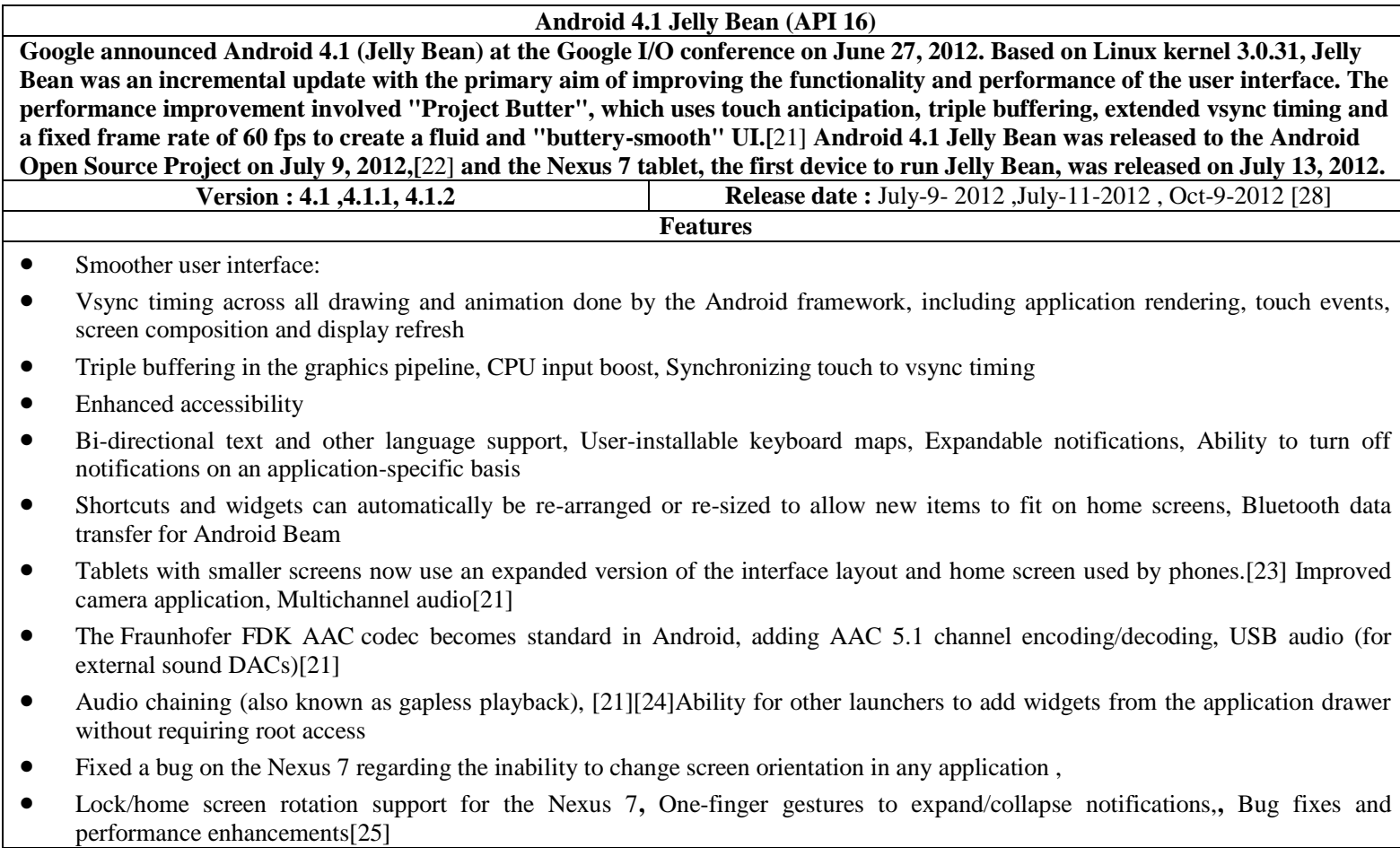

Android 4.2 Jelly Bean (API 17)

Google was expected to announce Jelly Bean 4.2 at an event in New York City on October 29, 2012, but the event was cancelled due to Hurricane Sandy.[26] Instead of rescheduling the live event, Google announced the new version with a press release, under the slogan "A new flavor of Jelly Bean". Jelly Bean 4.2 was based on Linux kernel 3.4.0, and debuted on Google's Nexus 4 and Nexus 10, which were released on November 13, 2012[27] \begin{tabular}{|l|l} 
Version : $4.2,4.2 .1,4.2 .2$ & Release date : November-13-2012, Nov 27,Feb-11-2013[29]
\end{tabular}

\section{Features}

- $\quad$ Lock screen improvements, including widget support and the ability to swipe directly to camera.[30]

- $\quad$ Notification power controls ("Quick Settings"), ,Daydream" screensavers, showing information when idle or docked

- Multiple user accounts (tablets only), Rewritten Bluetooth stack, switching from Bluez to Broadcom open source Blue Droid,[31] allowing improved support for multiple displays and wireless display (Miracast)

- $\quad$ Native right-to-left, always-on VPN and application verification.[32] A new NFC stack was added at the same time.[31]

- Accessibility improvements: triple-tap to magnify the entire screen, pan and zoom with two fingers. Speech output and Gesture Mode navigation for blind users, New clock application with built-in world clock, stop watch and timer 
- All devices now use the same interface layout, previously adapted from phones on 4.1 for smaller tablets (with centered software buttons, the system bar at the top of the screen, and a home screen with a dock and centered application menu), regardless of screen size

- Increased number of extended notifications and Actionable Notifications for more applications, allowing users to respond to certain notifications within the notification bar and without launching the application directly ,SE Linux ,Premium SMS confirmation,[33] Group Messaging

- $\quad$ Fixed a bug in the People application where December was not displayed on the date selector when adding an event to a contact[34], Added Bluetooth gamepads and joysticks as supported HID (Human interface device)

- Fixed Bluetooth audio streaming bugs, [35] Long-pressing the Wi-Fi and Bluetooth icons in Quick Settings now toggles the on/off state

- New download notifications, which now shows the percentage and estimated time remaining for active application downloads, New sounds for wireless charging and low battery, New Gallery application animation allows faster loading, USB debug white list ,Bug fixes and performance enhancements

- $\quad$ Fixed a bug in the People application where December was not displayed on the date selector when adding an event to a contact, Added Bluetooth gamepads and joysticks as supported HID (Human interface device)

- Fixed Bluetooth audio streaming bugs, Long-pressing the Wi-Fi and Bluetooth icons in Quick Settings now toggles the on/off state

- New download notifications, which now shows the percentage and estimated time remaining for active application downloads

- New sounds for wireless charging and low battery

- New Gallery application animation allows faster loading, USB debug white list, Bug fixes and performance enhancements

Android 4.3 Jelly Bean (API 18)

Google released Jelly Bean 4.3 under the slogan "An even sweeter Jelly Bean" on July 24, 2013, during an event in San Francisco called "Breakfast with Sundar". Most Nexus devices received the update within a week, although the secondgeneration Nexus 7 tablet was the first device to officially ship with it. A minor bug fix update was released on August 22,[36] Version : 4.3,4.3.1 $\quad$ Release date : July-24-2013, October-3-2013[37]

\begin{tabular}{lll}
\hline Version : 4.3,4.3.1 & \multicolumn{1}{c}{ Release date : July-24-2013, October-3-2013[37] } \\
\hline & Features & \\
\hline
\end{tabular}

- Bluetooth low energy support, Bluetooth Audio/Video Remote Control Profile (AVRCP) 1.3 support, OpenGL ES 3.0 support, allowing for improved game graphics, Restricted access mode for new user profiles [38]

- File system write performance improvement by running fstrim command while device is idle,[39] Dial pad auto-complete in the Phone application[38]

- Volume for incoming calls (ringtone) and notification alerts is no longer adjustable separately

- Improvements to Photo Sphere, Reworked camera UI, previously introduced on Google Play edition Phones[40]

- Addition of "App Ops", a fine-grained application permissions control system (hidden by default)[41]

- SELinux enabled by default, $4 \mathrm{~K}$ resolution support, Numerous security updates, performance enhancements, and bug fixes[42]

- System-level support for geofencing and Wi-Fi scanning APIs, Background Wi-Fi location still runs even when Wi-Fi is turned off

- Developer logging and analyzing enhancements

- $\quad$ Added support for five more languages, Changed digital rights management (DRM) APIs

- Right-to-left (RTL) languages now supported ,[38]Clock in the status bar disappears if clock is selected as lock screen widget ,Native emoji support[43]

- $\quad$ Bug fixes and small tweaks for the Nexus 7 LTE

Android 4.4 KitKat (API 19)

Google announced Android 4.4 KitKat on September 3, 2013. Although initially under the "Key Lime Pie" ("KLP") codename, the name was changed because "very few people actually know the taste of a key lime pie." Some technology bloggers also expected the "Key Lime Pie" release to be Android 5.[44] KitKat debuted on Google's Nexus 5 on October 31, 2013, and was optimized to run on a greater range of devices than earlier Android versions, having 512 MB of RAM as a recommended minimum; those improvements were known as "Project Svelte" internally at Google. The required minimum amount of RAM available to Android is $340 \mathrm{MB}$, and all devices with less than $512 \mathrm{MB}$ of RAM must report themselves as "low RAM" devices[45] Version : $4.4,4.4 .1,4.4 .2,4.4 .2,4.4 .3,4.4 .3 \quad$ Release date : October-31-2013 ,Dec-5-2013, Dec-09, June-02-2014 June-19-2014[46][47][48]

- Refreshed interface with white elements instead of blue

- Clock no longer shows bold hours; all digits are thin. The H, M, and S markings for the stopwatch and timer have been removed, leaving just the numbers.

- Ability for applications to trigger translucency in the navigation and status bars[49]

- Ability for applications to use "immersive mode" to keep the navigation and status bars hidden while maintaining user interaction[50]

- Action overflow menu buttons are always visible, even on devices with a "Menu" key, which was officially deprecated by Android 4.0. [51]

- Restriction for applications when accessing external storage, except for their own directories

- Optimizations for performance on devices with lower specifications, including zRAM support and "low RAM" device API[45]

- Wireless printing capability 
- $\quad$ NFC host card emulation, enabling a device to replace smart cards[45]

- Web Views now based on Chromium engine (feature parity with Chrome for Android 30), Expanded functionality for notification listener services[45]

- Public API for developing and managing text messaging clients, Storage Access Framework, an API allowing apps to retrieve files in a consistent manner. As part of the framework, a new system file picker allows users to access files from various sources (including those exposed by apps, such as online storage services). , New framework for UI transitions[52]

- Sensor batching, step detector and counter APIs[45] ,Settings application now makes it possible to select default text messaging and home (launcher) application

- Audio tunneling, audio monitoring and loudness enhancer, Built-in screen recording feature (primarily for developers, as usage of $\mathrm{ADB}$ is required)

- Native infrared blaster API, Verified boot, Enforcing SELinux, Expanded accessibility APIs and system-level closed captioning settings[53]

- Android Runtime (ART) introduced as a new experimental application runtime environment, not enabled by default, as a replacement for the Dalvik virtual machine, Bluetooth Message Access Profile (MAP) support[54]

- Disabled access to battery statistics by third-party applications

- $\quad$ Settings application no longer uses a multi-pane layout on devices with larger screens, Wi-Fi and mobile data activity (TX/RX) indicators are moved to quick settings

- Disables text wrapping in the Web View browser component, Improvements to auto focus, white balance and HDR+ for the Nexus 5 camera[55]

- Better application compatibility for the experimental Android Runtime (ART)

- Camera application now loads Google+ Photos instead of Gallery when swiping away from the camera view ,Miscellaneous improvements and bug fixes

- $\quad$ Further security enhancements and bug fixes ,

- Removal of the "App Ops" application permissions control system, introduced in Android 4.3, Refreshed Dialer app interface[56]

- Updated the Chromium-based Web View to version 33 (screen casting to DevTools, HTML5 Canvas hardware acceleration performance improvements, vibration API, HTML5 form validation, HTML5 data list) ,[57] Miscellaneous improvements and bug fixes

- $\quad$ CVE-2014-0224 fixed, eliminating an OpenSSL man-in-the-middle vulnerability[58]

\begin{tabular}{|l|l|}
\hline \multicolumn{2}{|c|}{ Android 4.4W KitKat, with wearable extensions (API 20) } \\
\hline On June 24, 2014, a version of Android KitKat exclusive to Android Wear devices was released. \\
\hline \multicolumn{3}{|c|}{ Version : 4.4W, 4.4W.1,4.4W.2 } & $\begin{array}{l}\text { Release date : June-25-2014, September-06-2014, October-21- } \\
2014[59][60]\end{array}$ \\
\hline \multicolumn{3}{|c|}{ Features } \\
\hline - $\quad \begin{array}{l}\text { Initial release of Android Wear platform for smart watches: the same as Android 4.4 "KitKat", but with wearable extensions } \\
\text { added }\end{array}$ \\
$\begin{array}{l}\text { UI updates for Google Maps navigation and alarms } \\
\text { Offline music playback, GPS support }\end{array}$
\end{tabular}

Android 5.0 Lollipop (API 21)

Android 5.0 "Lollipop" was unveiled under the codename "Android $L "$ on June 25, 2014, during Google I/O. It became available as official over-the-air (OTA) updates on November 12, 2014, for select devices that run distributions of Android serviced by Google, including Nexus and Google Play edition devices. Its source code was made available on November 3, 2014. [61]

Lollipop features a redesigned user interface built around a responsive design language referred to as "material design". Other changes include improvements to the notifications, which can be accessed from the lock screen and displayed within applications as top-of-the-screen banners. Furthermore, Google made internal changes to the platform, with the Android Runtime (ART) officially replacing Dalvik for improved application performance, and with changes intended to improve and optimize battery usage, known internally as Project Volta[62][63] \begin{tabular}{|l|l}
\hline Version : $5.0,5.0 .1,5.0 .2$ & Release date : November-12-2014, December-02-2014, December-
\end{tabular} $19-2014[64]$ Features

- Android Runtime (ART) with ahead-of-time (AOT) compilation and improved garbage collection (GC), replacing Dalvik that combines byte code interpretation with trace-based just-in-time (JIT) compilation [65] ,Support for 64-bit CPUs

- OpenGL ES 3.1 and Android Extension Pack (AEP) on supported GPU configurations

- Recent activities screen with tasks instead of applications, up to a configured maximum of tasks per application

- Vector drawables, which scale without losing definition, Support for print previews

- Material design, bringing a restyled user interface, Refreshed lock screen, no longer supporting widgets[66]

- Refreshed notification tray and quick settings pull-down

- Project Volta, for battery life improvements, Searches can be performed within the system settings for quicker access to particular settings[67]

- Lock screen provides shortcuts to application and notification settings, Guest logins and multiple user accounts are available on more devices, such as phones. [68]

- Audio input and output through USB devices 
- Third-party applications regain the ability to read and modify data located anywhere on external storage, such as on SD cards[69]. Pinning of an application's screen for restricted user activity.[67] Recently used applications are remembered even after restarting the device. [67]

- Web Views receive updates independently through Google Play for security reasons, instead of relying on system-wide vendor updates[70]

- Addition of 15 new languages: Basque, Bengali, Burmese, Chinese (Hong Kong), Galician, Icelandic, Kannada, Kyrgyz, Macedonian, Malayalam, Marathi, Nepali, Sinhala, Tamil and Telugu[71]

- Tap and Go allows users to quickly migrate to a new Android device, using NFC and Bluetooth to transfer Google Account details, configuration settings, user data and installed applications[67]

- A flashlight-style application is included, working on supported devices with a camera flash.[67] User-customizable priorities for application notifications., Smart lock feature,[72] SELinux in enforcing mode for all domains

- Updated emoji, Improved accessibility support (e.g. switch access support), Block-based over-the-air (OTA) updates for new device[73]

- A few bug fixes, including resolving issues with video playback and password failures handling

- Fixes a bug with TRIM support, which prevented devices from the nightly on-charger cleanups of file system allocations if the device was turned off while being charged, or if it was charged during the day.

- Changes how alarms wake the CPU, and how alarms compete for system resources[74]

\begin{tabular}{|c|c|}
\hline \multicolumn{2}{|c|}{ Android 5.1 Lollipop (API 22) } \\
\hline Version : $5.1,5.1 .1$ & Release date : March-09-2015,April-21-2015[75] \\
\hline \multicolumn{2}{|c|}{ Features } \\
\hline
\end{tabular}

- Improvements and bug-fixes to the Overview screen, Ability to join Wi-Fi networks and control paired Bluetooth devices from quick settings, Official support for multiple SIM cards[76]

- Device protection: if a device is lost or stolen it will remain locked until the owner signs into their Google account, even if the device is reset to factory settings.

- High-definition voice calls, available between compatible 4G LTE devices running Android 5.1, Improvements to the notification priority system, to more closely replicate the silent mode that was removed in Android 5.0[76][77]

- $\quad$ Various bug fixes, Native Wi-Fi support[78]

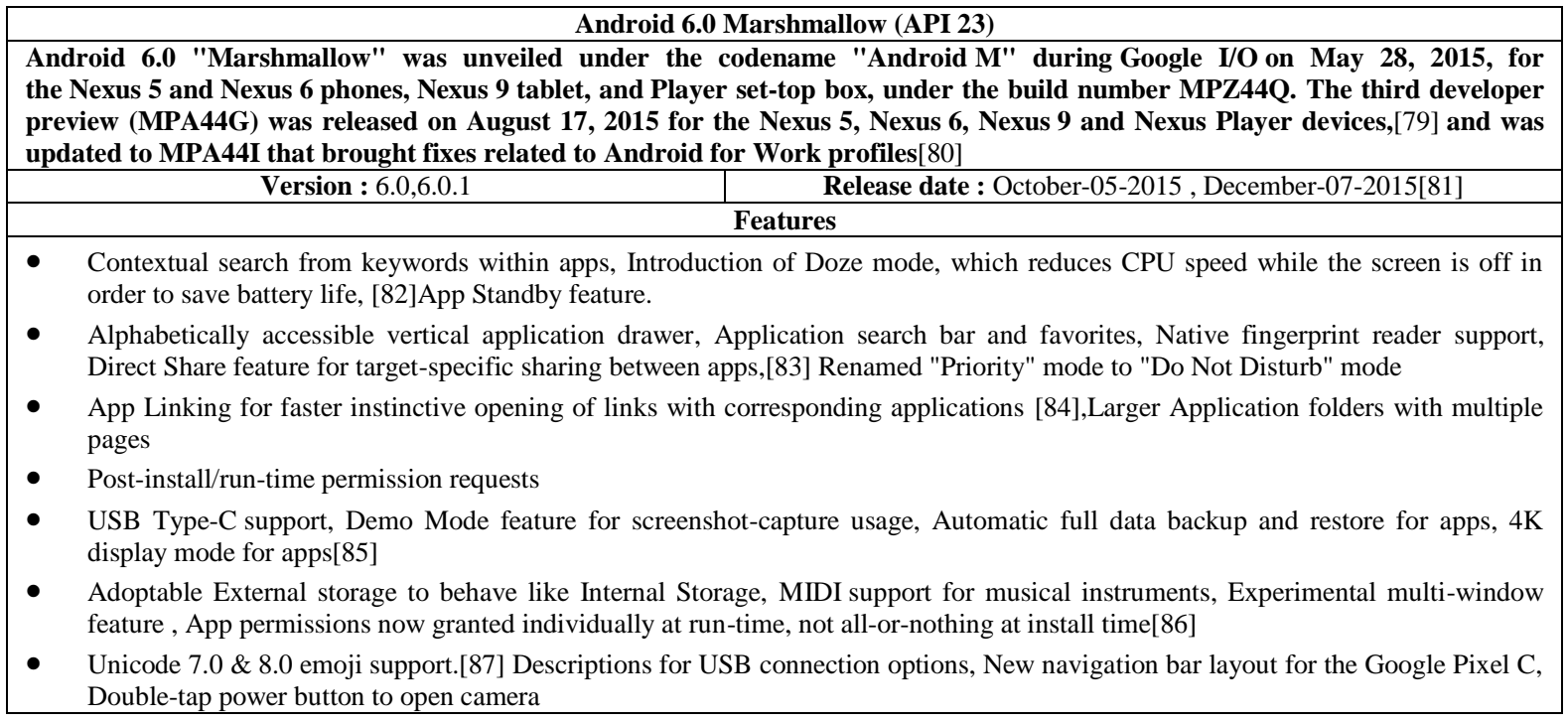

\section{Additional advance Features Includes In Android Nougat}

\section{- User experience}

Android Nougat introduces a split-screen display mode for phones, in which two apps can be snapped to occupy halves of the screen. An experimental multi-window mode is also available as a hidden feature, where multiple apps can appear simultaneously on the screen in overlapping windows. [88]

The notification shade was redesigned, featuring a smaller row of icons for settings, replacing notification cards with a new "sheet" design, and allowing inline replies to notifications (this feature is implemented via existing APIs that are used for similar functionality on Android Wear). Multiple notifications from a single app can also be "bundled",[89] and there is greater per-app control over notifications[90]

The "Doze" power saving mechanism introduced in Android Marshmallow was expanded to include a state activated when the device is running on battery and the screen has been off for a period of time but is not stationary. In this state, network activity is restricted, and apps are granted "maintenance windows" in which they can access the network and perform background tasks. As in Marshmallow, the full Doze state is activated if the device is stationary with its screen off for a period of time.[89][91] A new "Data Saver" mode restricts 
background mobile data usage, and can trigger internal functions in apps that are designed to reduce bandwidth usage, such as capping the quality of streaming media[91]

\section{- Platform}

In December 2015, Google announced that Android Nougat would switch its JRE (Java Runtime Environment) from the defunct Apache Harmony to OpenJDK - the official open source implementation of the Java platform maintained by Oracle Corporation and the Java community.[92]The Android Runtime (ART) now incorporates a profile-guided compilation system, utilizing a JIT compiler and profiling alongside its current ahead-of-time compiler to further optimize apps for a device's hardware and other conditions in the background.[89]

Nougat introduces a system for enabling "seamless", automatic system updates, based upon and sharing some code with the implementation of similar functionality on Chrome OS. The system uses a pair of SquashFS partitions; the Android system executes from an "online" partition, while updates are applied in the background to a redundant "offline" partition. On the next boot following the installation of an update, the redundant partition is designated as active, and the device henceforth boots into the updated system. The previous system partition is kept as a backup in case of update failure, and to serve as the "offline" partition for the next update. This system removes the requirement for the device to reboot into the system recovery environment to apply the update (which prevents the device from being used until the update is complete) and also provides the ability for an update to be automatically rolled back in case of a failure. Due to the partitioning requirements of this system, existing devices will not support seamless updates. Additionally, due to the ART changes on Nougat, apps no longer need to be re-compiled upon the first boot after a system update.[93]

Developer Preview 2 added platform support for Vulkan,[94] the new low-level 3D rendering API to augment OpenGL ES but with higher graphics performance. Nougat is the first version featuring Unicode 9.0 support and comes with updated emoji plus support for emoji skin tones.[95] [96]

Android 7.1 adds native API support for implementing image keyboards; multi endpoint telephony; shortcut menus and rounded icon assets for apps on launchers; and support for the Google Daydream virtual reality platform.[97] The Google Daydream feature is a specific "VR mode", with advanced technology for reduced graphics latency,[98] a "sustained performance mode" to assist developers in optimizing apps to a devices thermal profile,[99] a new head tracking algorithm which combines the input from various device sensors, and integration of system notifications into the VR user interface.[100]

\section{- Security}

In response to the Stage fright family of bugs disclosed and fixed in 2015, several changes were made to harden the media stack against future vulnerabilities. Runtime integer overflow detection was implemented, preventing the majority of Stage fright-like programming bugs from becoming vulnerabilities, [101] in addition to helping fix and prevent such bugs.[101]Android's monolithic Media Server process was redesigned to better adhere to the principle of least privilege. Media Server is now split into several separate processes, each running in its own unprivileged sandbox, and granted only the permissions required for its task. For example, only the Audio Server can access Bluetooth, and libstagefright now runs within the MediaCodecService sandbox, which is only granted GPU access. Further constraints were placed on the media stack through seccomp.[101]

Various mechanisms were enabled to reduce the possibility of malicious code being injected and/or executed inside the Linux kernel, including dividing kernel memory into logical segments for code and data, with page access permissions of read-only and no-execute as appropriate. The kernel was also restricted from directly accessing user space memory, and stronger stack protection was enabled in the GCC compiler to reduce stack smashing. To limit exposure of the kernel to potentially malicious code, perf was disabled by default, IOCtl commands were restricted by SELinux, and seccomp-bpf was enabled to grant processes the ability to restrict system calls.[102]

On devices shipping with Android Nougat, the "Verified Boot" policy (introduced partially on KitKat, and displaying notifications on startup on Marshmallow) must be strictly enforced. If system files are corrupted or otherwise modified, the operating system will only allow operation in a limited-use mode or refuse to boot at all.[103]

\section{About Release Procedure}

Android 7.0 was officially released on August 22, 2016, with the Nexus 6, Nexus 5X, Nexus 6P, Nexus 9, Nexus Player, Pixel C and General Mobile 4G as the first devices to receive the update.[104] Dave Burke, Android's Vice President of Engineering, stated in August 2016 that updates to Nougat would be released quarterly as maintenance releases focusing on "continued refinements and polish", with a new developer preview coming in the fall of 2016. On September 6, 2016, LG announced the V20, the first smart phone to ship 
with Nougat pre-loaded. Google unveiled the first-party Pixel and Pixel XL smart phones during a hardwarefocused event on October 4, 2016.[105]

Updates to existing devices will vary by manufacturer and carrier. HTC has stated that it planned to begin updating the HTC 10, HTC One A9 and HTC One M9 in the fourth quarter of 2016, and the Nougat update was subsequently released to unlocked handsets on November 25, 2016.[106] Sony also confirmed that it would update a range of its recent devices to Nougat. Motorola Updated their Moto G4 Plus from Android Marshmallow to Android Nougat Recently One Plus released Nougat with Oxygen OS 4 to the 3 and 3T models on December 31, 2016.[107]

Qualcomm stated that it would not support Nougat on devices using its Snapdragon 800 and 801 system-on-chips for undisclosed reasons. Although developer preview builds of Nougat were released for the device, Sony stated that it would not upgrade the Xperia Z3 (which uses the Snapdragon 801) to the final version due to "unforeseen platform limitations". It was reported that the Google Compatibility Test Suite (whose tests must be passed in order to receive official certification) specified that all devices running Nougat must support either Vulkan or OpenGL ES 3.1 graphics APIs neither of which are supported by the device's Adreno 330 graphics core.[108]

V. Hardware requirements And Software for Android operating system

\begin{tabular}{|c|c|c|}
\hline Hardware requirement[109] & \multicolumn{2}{|r|}{ Software requirement[109] } \\
\hline \multirow{3}{*}{$\begin{array}{l}\text { A 64-bit environment is required for } \\
\text { Gingerbread }(2.3 . \mathrm{x}) \text { and newer } \\
\text { versions, including the master branch. } \\
\text { the older versions are compile on 32- } \\
\text { bit systems. }\end{array}$} & \multicolumn{2}{|r|}{ Operating system } \\
\hline & GNU/Linux & Mac OS (Intel/x86) \\
\hline & \multirow{2}{*}{$\begin{array}{l}\text { Android } 6.0 \text { (Marshmallow) - } \\
\text { AOSP master: Ubuntu } 14.04 \\
\text { (Trusty) }\end{array}$} & \multirow[t]{2}{*}{$\begin{array}{l}\text { Android } 6.0 \text { (Marshmallow) - AOSP master: Mac OS } \\
\text { v10.10 (Yosemite) or later with Xcode } 4.5 .2\end{array}$} \\
\hline \multirow{2}{*}{$\begin{array}{l}\text { If running Linux in a virtual machine } \\
\text { then need at least } 16 \mathrm{~GB} \text { of } \\
\text { RAM/swap. }\end{array}$} & & \\
\hline & \multirow[t]{2}{*}{$\begin{array}{l}\text { Android 2.3.x (Gingerbread) - } \\
\text { Android 5.x (Lollipop): } \\
\text { Ubuntu 12.04 (Precise) }\end{array}$} & \multirow[t]{2}{*}{$\begin{array}{l}\text { Android 5.x (Lollipop): Mac OS v10.8 (Mountain Lion) } \\
\text { with Xcode 4.5.2 and Command Line Tools }\end{array}$} \\
\hline \multirow{3}{*}{$\begin{array}{l}\text { At least } 100 \mathrm{~GB} \text { of free disk space for } \\
\text { a checkout, } 150 \mathrm{~GB} \text { for a single build, } \\
\text { and } 200 \mathrm{~GB} \text { or more for multiple } \\
\text { builds. }\end{array}$} & & \\
\hline & \multirow[t]{2}{*}{$\begin{array}{l}\text { Android 1.5 (Cupcake) - } \\
\text { Android 2.2.x (Froyo): Ubuntu } \\
10.04 \text { (Lucid) }\end{array}$} & $\begin{array}{l}\text { Android 4.1.x-4.3.x (Jelly Bean) - Android 4.4.x (KitKat): } \\
\text { Mac OS v10.6 (Snow Leopard) or Mac OS X v10.7 (Lion) } \\
\text { and Xcode 4.2 (Apple's Developer Tools) }\end{array}$ \\
\hline & & $\begin{array}{l}\text { Android } 1.5 \text { (Cupcake) - Android 4.0.x (Ice Cream } \\
\text { Sandwich): Mac OS v10.5 (Leopard) or Mac OS X v10.6 } \\
\text { (Snow Leopard) and the Mac OS X v10.5 SDK }\end{array}$ \\
\hline
\end{tabular}

\section{Conclusion}

In this paper, explains about the android versions and also shown architecture details and how to manage hardware in android operating system and also some details about previous android versions and mainly this research about the latest version of android that is android 7.0 Nougat and also about its features and details about its release. Android Nougat is that version of android developed by Google which recovers the all drawbacks of marshmallow and also it contains extended features.

\section{References}

[1]. Ali Waqas (September 28, 2011). "Android 2.3.6 Gingerbread Update For Nexus S Available (Wi-Fi And Tethering Fix)". Addictive Tips. Retrieved December 20, 2011. "Introducing \#Android Nougat. Thank you, world, for all your sweet name ideas! \#Android Reveal". Twitter. June 30, 2016. Retrieved June 30, 2016.

[2]. Elgin, Ben (August 17, 2005). "Google Buys Android for Its Mobile Arsenal". Bloomberg Business week. Bloomberg. Archived from the original on February 24, 2011. Retrieved February 20, 2012.android architecture figure and explanation from "TECHBLOGON.COM"site given.

[3]. Amadeo, Ron (March 10, 2016). "Surprise! The Android N Developer Preview is out right now". Ars Technica. Condé Nast. Retrieved July 1, 2016..

[4]. Burke, Dave (April 13, 2016). "Android N Developer Preview 2, out today!" Android Developers blog. Google Inc. Retrieved June 5,2016

[5]. Savov, Vlad (May 18, 2016). "Google details Android N features ahead of late summer release". The Verge. Vox Media. Retrieved July 1, 2016. Kastrenakes, Jacob (May 18, 2016). "Google's latest Android N beta is meant for everyone". The Verge. Vox Media. Retrieved July 1, 2016.

[6]. "Google launches Android N Developer Preview 3 with seamless updates and VR mode". Venture Beat. Retrieved 9 July 2016.

[7]. Burke, Dave (June 15, 2016). "Android N APIs are now final, get your apps ready for Android N!". Android Developers blog. Google Inc. Retrieved July 1, 2016. Serrafero, Mario Tomas (June 15, 2016). "Android N Developer Preview 4 is out! Final APIs and Play Publishing". XDA Developers. Retrieved July 1, 2016.

[8]. "Android 7.0 Nougat Developer Preview 5 - the final preview - is available for download". Android Police. 2016-07-18. Retrieved 2016-07-19.

[9]. "Android N name revealed: It's Nougat". PC World. IDG. Retrieved 30 June2016. "Android 7.0 Nougat statue unveiled by Google". Phone Arena. Retrieved 30 June 2016. Gibbs, Samuel (July 1, 2016). "Nougat: Google's new Android name divides opinion". The Guardian. Retrieved July 30, 2016.

[10]. "Android 7.0 Nougat: a more powerful OS, made for you". Android Developers Blog. August 22, 2016. Retrieved August 23, 2016.

[11]. Lumb, David (October 11, 2016). "Download Android 7.1 Nougat in beta later this month". Engadget. Retrieved December 7, 2016. 
[12]. Fonts, Agustin (December 5, 2016). "A sweet update to Nougat: Android 7.1.1". The Keyword Google Blog. Google. Retrieved December 7, 2016. Ingraham, Nathan (December 5, 2016). "Android 7.1.1 is rolling out now". Engadget. Retrieved December 7, 2016.

[13]. Carman, Ashley (December 5, 2016). "Google is bringing Pixel features to its Nexus line with Android 7.1.1". The Verge. Vox Media. Retrieved December 7, 2016.

[14]. Maroger (October 19, 2011). "Ice Cream Sandwich Runs on Linux Kernel 3.0.1". Fine Oils. BlogSpot. Retrieved July 24, 2013, Rey, Francis (October 19, 2011). "Android 4.0 Ice Cream Sandwich SDK Now Available". Social Barrel. Retrieved July 24, 2013,Stevens, Tim (October 19, 2011). "Google confirms Nexus S will get Ice Cream Sandwich - for real this time (Gingerbread devices, too)". Engadget. Retrieved July 24, 2013.

[15]. Messina, Vincent (November 15, 2011). "Ice Cream Sandwich source code released, custom ROMs inevitable". Android Guys. Retrieved July 24, 2013, "Today Is The Last Day To Get Flash For Android". WebProNews. August 14, 2012. Retrieved February 7, 2013.

[16]. Molen, Brad (October 19, 2011). "Android 4.0 Ice Cream Sandwich now official, includes revamped design, enhancements galore". Engadget. Retrieved October 19, 2011, German, Kent (October 18, 2011). "Ice Cream Sandwich adds tons of new features". CNET. Retrieved July 24, 2013, Velazco, Chris (October 18, 2011). "A Quick Android 4.0 Ice Cream Sandwich Feature Rundown". Tech Crunch. Retrieved July 24, 2013.

[17]. Fitzpatrick, Jason. "What Exactly Happens When You Swipe An Android App From the Recent Apps List?". How-To Geek. Retrieved September 19, 2014,

[18]. "Android Supported Media Formats". Android Developers. Retrieved March 30, 2012, Kennemer, Quentyn (October 19, 2011). "Android 4.0 Ice Cream Sandwich has Hardware Acceleration". Phandroid. Retrieved October 19, 2011, "Wi-Fi Direct". Android Developers. Retrieved July 27, 2013.

[19]. Google announces 4.0.4 on Google+. March 28, 2012. Retrieved March 31, 2012.

[20]. "Android 4.0.3 Platform and Updated SDK tools". Android Developers Blog. December 16, 2011. Retrieved January 4, 2012,Google announces 4.0.4 on Google+. March 28, 2012. Retrieved March 31, 2012.

[21]. "Android 4.1 for Developers". Android Developers. Retrieved February 9,2013.

[22]. Queru, Jean-Baptiste. "Android 4.1 in AOSP". Retrieved July 12, 2012.

[23]. "Confirmed: Android 4.1 uses different layouts for different tablet sizes". ComputerWorld. Retrieved July 8, 2012.

[24]. "Issue 3461: Implement Gapless Playback of consecutive audio files". code.google.com. Retrieved November 12, 2012, "Android 4.1 finally adds gapless music playback to stock music apps". Pocketables.com. June 29, 2012. Retrieved June 30, 2012.

[25]. "Android 4.1.2 brings homescreen rotation to devices". GigaOM. October 9, 2012. Retrieved October 9, 2012, "New Feature In Android 4.1.2: Expanding/Collapsing Notifications With One Finger (Video)". Android Police. October 9, 2012. Retrieved October 9, 2012.

[26]. "Monday's Google event in NYC canceled due to Hurricane Sandy". Android Central. October 27, 2012. Retrieved February $19,2014$.

[27]. "Android 4.2 Jelly Bean lands on Nexus 7". Know Your Mobile. November 14, 2012. "Google Releases Nexus 4, Nexus 10 and Android 4.2". IGN. November 13, 2012. Retrieved November 16, 2012.

[28]. "Android 4.1.1 Nexus 7 Update". GSMArena.com. July 11, 2012. Retrieved February 26, 2013,"Android 4.1.2 rolling out for Nexus 7". The Verge. October 9, 2012. Retrieved October 9, 2012

[29]. "Android 4.2 adds gestue typing, wireless TV display, multiple user support on tablets, and more". The Verge. October $29,2012$. Retrieved October 29, 2012,"(Changelog) What's New In Android 4.2.1 (JOP40D)". Android Police. November 27, 2012. Retrieved November 27, 2012,"Breaking: Android 4.2.2 (Build JDQ39) Update Rolling Out To GSM Galaxy Nexus, Nexus 7, Nexus 10". Android Police. February 11, 2013. Retrieved February 12, 2013.

[30]. "Android 4.2 Jelly Bean brings all-new photography powers". TechRadar.com. October 29, 2012. Retrieved November 12, 2012.

[31]. Klug, Brian (May 19, 2013). "The Next Version of Android - Some of What's Coming". anandtech.com. Retrieved October 28, 2014.

[32]. "Jelly Bean". Android developer portal. Retrieved July 2, 2014

[33]. "Exclusive Android 4.2 Alpha Teardown, Part 2: SELinux, VPN Lockdown, And Premium SMS Confirmation". Android Police. October 17, 2012. Retrieved November 12, 2012.

[34]. "Sorry, Santa - Google Cancels December In Android 4.2". Android Police. November 17, 2012. Retrieved November $27,2012$.

[35]. "Android 4.2.2 brings Bluetooth audio fixes to Nexus phones and tablets". Ars Technica. February 12, 2013. Retrieved February $12,2013$.

[36]. "Google Announces Android 4.3, Update Rolling Out Today". Tom's Hardware. July 24, 2013. Retrieved July 25, 2013, "Android Open Source Project now has latest 4.3 fixes for most Nexus hardware". Engadget. August 22, 2013. Retrieved August $22,2013$.

[37]. "Android 4.3 announced, rolling out to Nexus devices today". The Verge. July 24, 2013. Retrieved July 24, 2013, "Suddenly, A Wild Android 4.3.1 Appears - LTE Nexus 7 Receiving Android 4.3.1 OTA (JLS36I)". Android Police. October 3, 2013. Retrieved October 4, 2013

[38]. "Introducing Android 4.3, a sweeter Jelly Bean". Official Android Blog. July 24, 2013. Retrieved July $30,2013$.

[39]. "Android 4.3 supports TRIM, improves performance on Nexus devices". Engadget. July 30, 2013. Retrieved July 30, 2013.

[40]. "Android KitKat". Android Developers Portal. android.com. Retrieved November 16, 2013, "Android on Intel Architecture". 01.org. July 11, 2013. Retrieved February 9,2014

[41]. "App Ops: Android 4.3's Hidden App Permission Manager, Control Permissions For Individual Apps!". Android Police. July 25, 2013. Retrieved July 30, 2013.

[42]. "Android 4.3 source code reveals support for 4K resolution". Engadget. July 26, 2013. Retrieved July 30, 2013, "An In-Depth Look At The Big (And Small) Additions To Android Jelly Bean 4.3". Android Police. July 24, 2013. Retrieved July $25,2013$.

[43]. Jaymar Cabebe (July 25, 2013). "Google Android 4.3 Jelly Bean Release Date, Price and Specs - CNET". CNET. CBS Interactive.

[44]. Kelion, Leo (September 3, 2013). "Android KitKat announced". BBC News. Retrieved September 3, 2013 , "Android 5.0 Key Lime Pie release date, news and rumors". TechRadar. July 2, 2013. Retrieved July 22, 2013.

[45]. Molen, Brad (November 5, 2013). "Engadget review the Nexus 5". Engadget. Retrieved November 11, 2013, "7.6.1". Android Compatibility Definition Document (PDF) (4.4 ed.). Google. November 27, 2013. p. 33.

[46]. "Android for all and the new Nexus 5 ". Google Official Blog. October 31, 2013. Retrieved November 1, 2013, Android Developers Portal. Retrieved November 1, 2013.

[47]. Official Nexus Google+ page (December 5, 2013). "Android 4.4.1 Released to Nexus 5". Google Plus. Retrieved December 5, 2013, Artem Russakovskii (December 9, 2013). "Android 4.4.2 (KOT49H) Is Already Rolling Out To All Nexus Devices Here Are The OTA ZIP Links For Manual Updating". Android Police. Retrieved December 9, 2013 
[48]. Ron Amadeo (June 2, 2014). "Google releases Android 4.4.3 to Nexus devices". Ars Technica. Retrieved June 2, 2014. Kellex (June 19, 2014). "Whoa: Android 4.4.4 Factory Images Posted as Build KTU84P". Droid Life. Retrieved June 19, 2014.

[49]. Bonn, Dieter (October 31, 2013). "Google's Nexus 5 with KitKat available today, starting at \$349: hands-on impressions". The Verge. Retrieved November 1,2013.

[50]. David Ruddock (October 31, 2013). "KitKat Feature Spotlight: Apps Can Now Go Truly Full-Screen, Hide The Navigation Bar". androidpolice.com. Retrieved November 3, 2013.

[51]. "Android menu button now on by default on all device with KitKat". PhoneArena.com. December 9, 2013. Retrieved February 9, 2014.

[52]. Ho, Joshua. "Examining MicroSD changes in Android 4.4". Anandtech. Retrieved March 28, 2014,"Getting Your SMS Apps Ready for KitKat". Android Developers Blog. Retrieved October 14, 2013.

[53]. "Android 4.4 brings support for low-power audio playback". PhoneArena.com. October 31, 2013. Retrieved February 25, 2014, Jason Cipriani (November 5, 2013). "How to record your screen on Android 4.4 KitKat". CNET. CBS Interactive. Retrieved January 4, 2014.

[54]. "Android Developers: ART and Dalvik". source.android.com. March 9, 2015. Retrieved March 19, 2015, David Ruddock (October 31, 2013). "KitKat Feature Spotlight: Android Finally Supports Bluetooth MAP, Will Make Bluetooth Integration With Your Car Suck A Lot Less". Android Police. Retrieved November 2, 2013.

[55]. "Android Issue 62378: KitKat WebView text wrap no longer works". code.google.com. Retrieved October 28, 2014, Christopher Trout (December 5, 2013). "Android update focuses on Nexus 5 camera improvements". Engadget. Retrieved December 5, 2013, David Pierce (December 5, 2013). "Fixing the Nexus 5: with a new version of Android, Google tackles the camera". The Verge. Retrieved December 6, 2013.

[56]. Peter Eckersley (December 12, 2013). "Google Removes Vital Privacy Feature From Android, Claiming Its Release Was Accidental". Electronic Frontier Foundation. Retrieved December 14, 2013, Kellex (June 2, 2014). "Fresh New Dialer Arrives in Android 4.4.3". Droid Life. Retrieved June 2, 2014.

[57]. Android Developers (June 3, 2014). "Android 4.4.3 aka KitKat MR2 is rolling out - Google+". Android Developers (Google+). Retrieved June 4, 2014.

[58]. Ryan Whitwam (June 19, 2014). "Google Rolling Out Android 4.4.4 Update (KTU84P) With A Security Fix, Factory Images/Binaries Up For Nexus Devices". androidpolice.com. Retrieved June 25, 2014

[59]. Rob, Triggs. "Google uploads part of the Android Wear source code to AOSP". Android Authority. Retrieved July 22, 2014, Andrew Grush (September 6, 2014). "Android Wear platform fully unveiled at I/O 2014". Android Authority. Retrieved January $25,2015$.

[60]. Michael Crider (September 6, 2014). "Samsung Gear Live Updated To Android Wear 4.4W.1 Build KGW42N: Here's What's New". Android Police. Retrieved January 25, 2015, Rita El Khoury (October 21, 2014). "Android Wear 4.4W.2 Build KNX01Q Starts Rolling Out For The LG G Watch, Brings Offline Music And GPS Support (Though You Can't Use The Latter)". Android Police. Retrieved January 25, 2015

[61]. "From Android L to Google Fit: All the new products and features from Google I/O". PC World. June 25, 2014. Retrieved June 26, 2014, "Updated Android Lollipop Developer Preview image coming to Nexus devices in a couple of days". PhoneArena.com. October 15, 2014. Retrieved October 16,2014.

[62]. "Google's new 'Material Design' UI coming to Android, Chrome OS and the web". Engadget. June 25, 2014. Retrieved June 26, 2014, "We just played with Android's L Developer Preview". Engadget. AOL. Retrieved June 26, 2014.

[63]. "Google Reveals Details About Android L at Google IO". Anandtech. Retrieved June 26, 2014,Andrei Frumusanu (July 1, 2014). "A Closer Look at Android RunTime (ART) in Android L". AnandTech. Retrieved July 5, 2014.

[64]. "Android 5.0 APIs". Android.com. Retrieved October 28, 2014. Liam Spradlin (December 2, 2014). "Android 5.0.1 (LRX22C) Hitting AOSP Now (Update: Factory Images Too)". Android Police. Retrieved December 3, 2014,"Factory Images for Nexus Android". Google.

[65]. "Android 5.0 APIs". Android.com. Retrieved October 28, 2014, Ben Cheng; Bill Buzbee (May 2010). "A JIT Compiler for Android's Dalvik VM"(PDF). android-app-developer.co.uk. Google. pp. 5-14. Retrieved March 19, 2015.

[66]. "Exploring Android L: Lockscreen widgets also bite the dust". Pocketables.com. June 2014. Retrieved January $25,2015$.

[67]. "Android 5.0 Lollipop's new features". India Times. November 27, 2014. Retrieved December 23, 2014.

[68]. "Lollipop brings proper multi-user accounts to your phone". Android Central. Retrieved January 25, 2015.

[69]. Cody Toombs (November 4, 2014). "Android 5.0 Makes SD Cards Great Again, Extends API To Allow Full Directory Access, Automatic MediaStore, And Improves Security". Android Police. Retrieved November 29, 2014,Michael Crider (November 27, 2014). "Root Explorer 3.3 Update Adds SD Card Write Capability Without Root In Lollipop". Android Police. Retrieved November 29,2014 .

[70]. "Lollipop stops Chromium bugs from endangering Android". ZDNet. October 24, 2014. Retrieved October 28,2014

[71]. $\quad$ "Android - 5.0 Lollipop". Android.com. November 3, 2014. Retrieved November 28, 2014.

[72]. Jason Cipriani (November 3, 2014). "Set up 'Priority' mode on Android 5.0 Lollipop". CNET. Retrieved December 23, 2014, "Smart lock screen security options in Android 5.0 Lollipop".

[73]. "Android 5.0 Emoji Changelog". Emojipedia. Retrieved 2 February 2017, "Accessibility features in Android 5.0 Lollipop". Android Central. October 25, 2014. Retrieved January 30, 2016. "Block-Based OTAs | Android Open Source Project". Source.android.com. Retrieved January 30, 2016.

[74]. Stephen Schenck (December 22, 2014). "What's actually new in Android 5.0.2?". PocketNow.com. Retrieved January 6, 2015.

[75]. David Nield. "10 Things You Can Do in Android 5.1 That You Couldn't Before". Field Guide. Gawker Media, "Android 5.1.1 (LMY47V) officially uploaded to AOSP and here's the full changelog, Nexus Player factory images also available". Retrieved May $1,2015$.

[76]. "Google confirms Android 5.1 is rolling out today, will support multi-SIM devices and HD calls". VentureBeat. Retrieved April $24,2015$.

[77]. "Android 5.1 reinstates Silent Mode, but there's a catch". AndroidPIT. Retrieved March 27, 2015.

[78]. "Android 5.1.1 (LMY47V) officially uploaded to AOSP and here's the full changelog, Nexus Player factory images also available". Retrieved May 1, 2015, Jennings, Jack (June 26, 2015). "Google Open Source Code for Wi-Fi Calling". XDA. Retrieved November $2,2015$.

[79]. "Google's Android M preview build will run on the Nexus 5, 6, 9, and Player [Updated]". Ars Technica. Condé Nast. Retrieved May 28, 2015, "Downloads Android Developers". August 17, 2015.

[80]. "Support and Release Notes | Android Developers". developer.android.com. Retrieved August 22, 2015. 
[81]. "Get ready for the sweet taste of Android 6.0 Marshmallow". Android Developers. Retrieved October 6, 2015, "Google releases Android 6.0.1 Factory Images for Nexus 6P/6, Nexus 5X/5, Nexus Player, Nexus 9 And Nexus 7 [2013]". GadgetzArena. Retrieved March 10,2016.

[82]. "Now on Tap", "Android Doze".

[83]. "Android vertical app drawer", "Android 6.0 APIs - Android Developers".

[84]. "Handling App Links". developer.android.com, "App Linking in Android 6.0". developer.android.com.

[85]. "Use Android 6.0's Hidden System UI Tuner to Customize Your Quick Settings and Status Bar","Android 6.0 APIs - Android Developers".

[86]. "MIDI - Android Open Source Project", "How to Unlock the Hidden Multi-Window Mode in Android 6.0 Marshmallow", "Enable Multi-Window mode in Android 6.0 Marshmallow with a bit tinkering",http://developer.android.com/training/permissions/requesting.html

[87]. "Android 6.0.1 Emoji Changelog". Emojipedia. Retrieved 2 February 2017.

[88]. Amadeo, Ron (March 21, 2016). "This is Android N's freeform window mode". Ars Technica. Condé Nast. Retrieved July 1, 2016.

[89]. Amadeo, Ron (March 10, 2016). "Surprise! The Android N Developer Preview is out right now". Ars Technica. Condé Nast. Retrieved July 1, 2016.

[90]. "Android Nougat release date: when you'll get it and why you'll want it". techradar. July 31, 2016. Retrieved August 9, 2016.

[91]. "Android N for Developers". Android Developers. Google Inc. Retrieved March 10, 2016, "Android: The Road to JIT/AOT Hybrid Compilation-Based Application User Experience". software.intel.com. Intel Corporation. Retrieved 2016-07-15.

[92]. Amadeo, Ron (January 7, 2016). "Android N switches to OpenJDK, Google tells Oracle it is protected by the GPL". Ars Technica. Condé Nast. Retrieved July 1,2016.

[93]. "Android N seamless updates not coming to existing devices". GSMArena. Retrieved 9 July 2016, "Android N borrows Chrome OS code for "seamless" update installation". Ars Technica. Retrieved 9 July 2016.

[94]. Burke, Dave (April 13, 2016). "Android N Developer Preview 2, out today!". Android Developers blog. Google Inc. Retrieved June 5, 2016. "Vulkan Graphics API". Android Developers. Google Inc. Retrieved July 1, 2016.

[95]. Woods, Shannon (April 13, 2016). "Optimize, Develop, and Debug with Vulkan Developer Tools". Android Developers blog. Google Inc. Retrieved July 1, 2016, "Support and Release Notes - Developer Preview 2". Android Developers. Google Inc. April 13, 2016. Retrieved April 14, 2016

[96]. "Android N Developer Preview 2, out today!". Android Developers Blog. Google. April 13, 2016. Retrieved August 5, 2016.

[97]. Lumb, David (October 11, 2016). "Download Android 7.1 Nougat in beta later this month". Engadget. Retrieved December 7, 2016.

[98]. "Gear VRs for everyone! Google turns Android into a VR-ready OS: Daydream". Ars Technica. Ars Technica. May 5, 2016. Retrieved August 4, 2016.

[99]. "Android N for Developers". Android Developers. Google Inc. Retrieved March 10, 2016.

[100]. "VR at Google - Google I/O 2016"". YouTube. Google. May 9, 2016. Retrieved August 4, 2016.

[101]. "Hardening the media stack". android-developers.blogspot.com. Google. Retrieved 2016-08-03.

[102]. "Protecting Android with more Linux kernel defenses". security.googleblog.com. Google. Retrieved 2016-08-03.

[103]. "Android Nougat won't boot your phone if its software is corrupt". Engadget. AOL. Retrieved 20 July 2016 ,"Android 7.0 devices could be harder to root, won't boot at all if the software is corrupt". PhoneArena. Retrieved 20 July 2016.

[104]. "Android 7.0 Nougat: a more powerful OS, made for you". Android Developers Blog. August 22, 2016.

[105]. Burke, Dave (August 22, 2016). "Taking the final wrapper off of Android 7.0 Nougat". Android Developers Blog. Retrieved August 24, 2016, "LG launches V20 Smartphone with Android 7.0 Nougat". The Verge. Retrieved 22 September 2016, "Google's new Pixel phones come with Android 7.1 Nougat". The Verge. Retrieved 4 October 2016.

[106]. Gunther, Cory (December 5, 2016). "HTC Android 7.0 Nougat Update Release Details". GottaBeMobile. Retrieved December 7, 2016.

[107]. O'Boyle, Britta; Hall, Chris (December 6, 2016)."When is Android 7.1.1 Nougat coming to my phone?". Pocket-lint. Retrieved December 7, 2016, Woods, Ben (December 31, 2016). "Android Nougat rolling out to OnePlus 3 and 3T from today". TrustedReviews. Retrieved December 31, 2016.

[108]. Cunningham, Andrew (August 31, 2016). "Why isn't your old phone getting Nougat? There's blame enough to go around". Ars Technica. Retrieved December 7, 2016, V. Cosmin (August 31, 2016). "Sony claims it's not at fault for not releasing Android 7.0 Nougat for Xperia Z3". PhoneArena. Retrieved December 7, 2016.

[109]. Refered from the site :http://source.android.com/source/requirements.html

[110]. Above figure no- 2 referd from site:http://en.wikipedia.org/wiki/android_version_history.

[111]. [A] All reference from :https://enwikipedia.org/wiki/Android_version_history

[112]. [B]All reference from : http://en.wikipedia.org/wiki/Android_Nougat 